\title{
Bemerkungen zur ökonomischen Theorie der Institutionenbildung
}

\section{Probleme der Theorie der Institutionenbildung}

Die Bildung von Institutionen ist ein Grundproblem menschlicher Kultur, das von verschiedenen Wissenschaftszweigen reflektiert wird. Dabei sind deskriptive Aussagen darüber, wie Institutionen tatsächlich entstehen von normativen ihres Legitimationsgrundes zu unterscheiden. Die Rechtswissenschaft neigt dazu, die normative Frage in den Vordergrund zu stellen, z.B. in der Staatslehre oder der Rechtsphilosophie. Aber auch empirische Blickwinkel sind ihr nicht fremd, z.B. in der Staatssoziologie oder Teilen der Governance-Forschung.

Die Frage der Bildung von Institutionen hört dabei nicht dadurch auf, von Interesse zu sein, dass es überhaupt ausgebildete Institutionen gibt. Eine Institutionentheorie ist nicht nur für einen imaginären Naturzustand oder eine zerfallene Gesellschaft von Interesse. Denn erstens ist die nationale und globale Ordnung institutionell keineswegs saturiert die bestehenden Strukturen müssen wegen erkannter Dysfunktionalitäten und neuer Herausforderungen weiter entwickelt werden. Die Föderalismusreform bildet ein Beispiel aus Deutschland, die Konstitutionalisierung Europas (oder ihr Ausbleiben, das ebenfalls institutionelle Veränderungen bringen würde) eines aus einem supranationalen Rechtskreis. Institutionen müssen aber nicht nur gebildet und weiterentwickelt, sondern auch erhalten werden. Auch dies wird nicht gelingen, wenn nicht ein klares Bewusstsein dafür besteht, wie es zur Institutionenbildung kommt. Die Untersuchung von Kosfeld/ Riedl liefert eine Analyse aus dem Blickwinkel der Behavioral Economics zu diesem Thema, die wie andere Analysen dieser Art deshalb ganze Aufmerksamkeit verdient.

Die sich anschließenden Bemerkungen werden - nach einer kurzen Zusammenfassung dessen, was genau Gegenstand dieses Kommentars sein soll - eine Beziehung entfernt, schwach, aber sichtbar - von Befunden der Behavioral Econocmis zu bestimmten Forschungen zur menschlichen moralischen Urteilskraft herstellen, die man in den modernen Kognitionswissenschaften findet und die traditionelle Erkenntnisse der Ethik aufgreifen. Sie werden dann einige Konsequenzen für eine Theorie der Institutionenbildung andeuten, die sich aus diesen Befunden ergeben könnten.

Als Kerngedanke der Untersuchung (wie anderer, an die sie anknüpft) kann gelten, dass es empirische Hinweise dafür gibt, dass Menschen Institutionen in einer Weise bilden, die spieltheoretischen Standardannahmen widerspricht: »(T)theoretical models based on common knowledge of rationality and egoistic preferences largely fail to predict actual behaviour $\ll$.

\section{Moralische Parameter der Institutionenbildung}

Die Untersuchung deutet an, dass es Agenten der Institutionenbildung nicht nur um Nutzenmaximierung in einem engen Sinn, sondern auch um andere Ziele geht. Bedeutsam ist dabei insbesondere die Inklusivität der geschaffenen Institution. ${ }^{1}$ Diese 

feld/Riedl

soll nicht nur so groß sein, dass sich für die Gründer der Institution ein Nutzen ergibt, sondern alle einschließen. Die Probanden zeigten Anzeichen von Abneigungen gegen Ungerechtigkeiten, die über unmittelbare Nutzenerwägungen hinausgehen. ${ }^{2}$

Dieser Befund hat aufgrund der Eigenart eines Experiments und der untersuchten Fragestellung bestimmte Grenzen und man sollte sich hier wie auch sonst vor Übergeneralisierung hüten. Jedenfalls kann man ihn aber als Ermutigung auffassen, über empirische Motivationslagen von Menschen ohne apriorische Setzungen nachzudenken. Das ist nicht selbstverständlich, denn es gibt in manchen Teilen der ökonomischen Theorie und von ihr inspiriert in der ökonomischen Analyse des Rechts die Tendenz, die menschliche Motivation für einfach und auf schlichte, kühle Nutzenmaximierung gerichtet anzusehen. Die methodische Anziehungskraft der Behavioural Economics ist ohne Zweifel ihre empirisches Orientierung, die Öffnung theoretischer Modelle für insbesondere psychologische Theorien, und die Bereitschaft, die theoretischen Modelle dem nützlichen Vergleich mit der menschlichen Realität auszusetzen. Dies gilt auch für eine entsprechend methodisch orientierte Analyse des Rechts. ${ }^{3}$ Was immer man von den konkreten Ergebnissen der Behavioural Economics und ihrer Anwendung auf das Recht im Einzelnen, auch bestimmten Aspekten ihrer Grundannahmen halten $\mathrm{mag}^{4}$ - dies ist ohne Zweifel ein Schritt nach vorn.

Es darf an diesem Punkt daran erinnert werden, dass die Ideengeschichte in ihren wesentlichen Teilen ganz selbstverständlich von komplexen Mechanismen der menschlichen Motivation und Entscheidungsfindung ausgegangen ist. Die Idee, das Menschen in einem engen Sinne nützlichkeitsmaximierend in Bezug auf ein bestimmtes Präferenzset agieren würden, ist deshalb im hohen Maße begründungsbedürftig.

Eine zentrale Rolle spielt bei einer komplexeren Theorie menschlicher Entscheidungsfindung die moralische Motivation - nicht in dem Sinne, dass Menschen notwendig gut und gerecht handelten - eine in Anbetracht der Erfahrung menschlicher Untaten selbst für die nachdrücklichsten Verfechter der Bedeutung der Moral wie etwa Kant wenig naheliegende These ${ }^{5}$-, sondern in dem Sinne, dass es jedenfalls moralische Einflüsse gebe, die das Entscheiden und Handeln manchmal auch tatsächlich bestimmten. Die moralische Orientierung wurde traditionell an ein bestimmtes menschliches Vermögen gebunden, von Sokrates Daimonion, über Thomas v. Aquins Synderesis, den Moral Sense der schottischen Aufklärer des 18. Jahrhunderts bis zu Kants praktischer Vernunft.

1 Vgl. dazu auch näher, Kosfeld/Okada/Riedl, Institution formation in public goods games, METEOR Discussion Pater Series, Maastricht, 2006.

2 Vgl. ebd.

3 Vgl. z.B. Jolls/Sunstein/Thaler, A Behavioural Approach to Law and Economics, in: Sunstein (ed.), Behavioral Law and Economics, 2000, p. 14: »The task of behavioural law and economics, simply stated, is to explore the implications of actual (not hypothesized) human behaviour for the law. How do »real people « differ from homo economicus?«

4 Vgl. z.B. Sunstein, Moral Heuristics, 28 Behavioral and Brain Sciences 531 (2005) zu bestimmten moralischen und aus seiner Sicht zweifelhaften Einflüssen auf Entscheidungen im Recht und die grundsätzlichen und kritischen Kommentare von Mikhail, Moral Heuristics or Moral Competence? Reflections on Sunstein, 28 Behavioral and Brain Sciences, 557 (2005) hierzu.

5 Kant, Idee zu einer allgemeinen Geschichte in weltbürgerlicher Absicht, Akademie Ausgabe, Bd. VIII, S. 23. 
Diese Realität moralischer Motivation wird interessanterweise in der Gegenwart mit den konzeptionellen Mitteln der modernen Theorie des menschlichen Geistes rekonstruiert, wobei durchaus pluralistische Ansätze aus dem breitgefächerten Spektrum der Kognitionswissenschaften gewählt werden. Die empirische Analyse der moralischen Urteilsfähigkeit wird so ein kognitionswissenschaftliches Anliegen, das für das Grundlagenverständnis von Ethik und Rechtswissenschaft große Bedeutung haben kann. ${ }^{6}$

Die interessante Frage, die damit aufgeworfen wird, ist die von Parallelen solcher Art der Grundlagenforschung der Ethik und der Theorie menschlicher Entscheidung der Behavioural Economics und ihrer Anwendung auf das Recht - eine Frage, die jedenfalls implizit auch am Horizont der internationalen Debatten aufzutauchen scheint. ${ }^{7}$ Jedenfalls fügen sich die Ergebnisse von Kosfeld/Riedl in dieses Bild ein und ermutigen durch ihre Substanz, in diese Richtung weiter mit theoretischer Phantasie und über Fachgrenzen hinweg nachzudenken.

\section{Konsequenzen für die Institutionentheorie}

Für die Theorie der Bildung, Weiterentwicklung oder Erhaltung von Institutionen (je nach Blickwinkel) ändert sich damit aus normativer Perspektive wenig. Wie immer menschliche Institutionenbildung faktisch sich vollziehen mag - über die Legitimation der gebildeten Institutionen ist damit nichts ausgesagt. Was faktisch geschieht, kann selbstverständlich normativ ein großes Übel sein.

Wenn es aber zutrifft, dass bei der Theorie der Institutionenbildung faktisch nicht nur schlichte Nutzenmaximierung wirkt, sondern auch andere Maßstäbe, vielleicht sogar so anspruchsvolle wie die der Gerechtigkeit, dann ist dies dennoch nicht bedeutungslos. Es kann als Hinweis darauf dienen, dass nicht nur das Hobbes'sche Muster der Interessenkalkulation bei der Institutionenbildung (und seine vielen theoretischen Nachfolger bis zur Spieltheorie) eine Rolle spielen, sondern etwas wie die Kantische Pflicht, in den bürgerlichen Zustand einzutreten, vielleicht tatsächlich von Menschen empfunden und zur Grundlage des Handelns gemacht wird. Dies ist dann in die Institutionentheorie zu integrieren, was auf verschiedene Weise geschehen kann. Etwa kann der Einwand einer pessimistischen philosophischen Anthropologie gegen bestimmte Institutionen wegfallen, dass diese unmöglich geschaffen werden könnten, weil sie durch ein Nutzenkalkül nicht rational rekonstruiert werden könnten. Positiv eröffnet sich die nicht unattraktive Perspektive einer normativ mutigeren, vielleicht sogar großzügigeren Konzeption menschlicher Institutionen. Um ein aktuelles Beispiel zu nennen: Die internationale Völkerrechtsordnung wird in der Gegenwart durch einen machtpolitischen Unilateralismus bedroht. Diese Lage wird unter verschiedenen Gesichtspunkten diskutiert, anklagend, enttäuscht, zynisch-resignierend. Der Befund, dass Menschen in ihrer empirischen Motivation, Institutionen zu bilden, nicht nur von

6 Vgl. z.B. im Überblick, Mahlmann/Mikhail, Cognitive Science, Ethics and Law, in: Z. Bankowski (Hrsg.): Epistemology and Ontology, 2005, S. 95ff.

7 Vgl. o. die Debatte in Fn 4. 
ihrem eigenen Nutzen geleitet werden, sondern auch von Maßstäben der Fairness und Gerechtigkeit, kann dazu ermutigen, das Projekt einer internationalen Friedensordnung nicht nur für normativ wünschenswert, sondern anthropologisch und entscheidungstheoretisch für möglich zu halten. Diese Art von theoretischer Ermutigung ist zugebener Maßen in Anbetracht der Großprobleme eines epochalen Projekts wie einer internationalen Rechtsordnung von bescheidener Bedeutung, aber immerhin Wert, nicht vergessen zu werden.

Insgesamt ergibt sich die Perspektive einer komplexen deskriptiven Theorie menschlicher Institutionen, gerade rechtlich konstituierter, deren Plausibilität mit ihrer empirischen Nähe zu einer überzeugenden und differenzierten Theorie menschlicher Motivation und Entscheidungsfindung steigt, in der normative Parameter eine Rolle spielen und nicht nur das kühle Nutzenkalkül des homo oeconomicus. 